\title{
Global analysis of cloud field coverage and radiative properties, using morphological methods and MODIS observations
}

\author{
R. Z. Bar-Or, O. Altaratz, and I. Koren \\ Department of Environmental Sciences and Energy Research, Weizmann Institute of Science, Rehovot, Israel
}

Received: 18 July 2010 - Published in Atmos. Chem. Phys. Discuss.: 19 August 2010

Revised: 25 November 2010 - Accepted: 17 December 2010 - Published: 11 January 2011

\begin{abstract}
The recently recognized continuous transition zone between detectable clouds and cloud-free atmosphere ("the twilight zone") is affected by undetectable clouds and humidified aerosol. In this study, we suggest to distinguish cloud fields (including the detectable clouds and the surrounding twilight zone) from cloud-free areas, which are not affected by clouds. For this classification, a robust and simple-to-implement cloud field masking algorithm which uses only the spatial distribution of clouds, is presented in detail. A global analysis, estimating Earth's cloud field coverage $\left(50^{\circ} \mathrm{S}-50^{\circ} \mathrm{N}\right)$ for 28 July 2008 , using the Moderate Resolution Imaging Spectroradiometer (MODIS) data, finds that while the declared cloud fraction is $51 \%$, the global cloud field coverage reaches $88 \%$. The results reveal the low likelihood for finding a cloud-free pixel and suggest that this likelihood may decrease as the pixel size becomes larger. A global latitudinal analysis of cloud fields finds that unlike oceans, which are more uniformly covered by cloud fields, land areas located under the subsidence zones of the Hadley cell (the desert belts), contain proper areas for investigating cloud-free atmosphere as there is $40-80 \%$ probability to detect clear sky over them. Usually these golden-pixels, with higher likelihood to be free of clouds, are over deserts. Independent global statistical analysis, using MODIS aerosol and cloud products, reveals a sharp exponential decay of the global mean aerosol optical depth (AOD) as a function of the distance from the nearest detectable cloud, both above ocean and land. Similar statistical analysis finds an exponential growth of mean aerosol fine-mode fraction (FMF) over oceans when the distance from the nearest cloud increases. A $30 \mathrm{~km}$ scale break clearly appears in several analyses here,
\end{abstract}

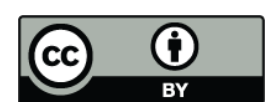

Correspondence to: I. Koren (ilan.koren@weizmann.ac.il) suggesting this is a typical natural scale of cloud fields. This work shows different microphysical and optical properties of cloud fields, urging to separately investigate cloud fields and cloud-free atmosphere in future climate research.

\section{Introduction}

Clouds and aerosols play key roles in the climate system as major components in the Earth's energy system and water cycle (Kiehl and Trenberth, 1997; Trenberth et al., 2009). The growing usage of space-borne platforms as remote sensing tools for cloud and aerosol research during the last decades (Kaufman et al., 2002; King et al., 2003) raised the need to develop accurate methods to retrieve cloud and aerosol properties (Platnick et al., 2003; Remer et al., 2005; Levy et al., 2007). In order to do so it is common to classify the imaged domain into two: detectable cloud and "assumed to be" cloud-free, in order to retrieve cloud and aerosol properties separately. For this purpose a wide range of cloud detection and masking algorithms has been developed. The majority of these algorithms use threshold techniques which separate cloud from cloud-free atmosphere by their reflectance values in different wavelengths or their variances; the cloud masking algorithms may also use different thresholds or techniques according the final product they serve (Ackerman et al., 1998; Platnick et al., 2003; Dybbroe et al., 2005; Luo et al., 2008). However, it was recently shown that there is a smooth transition from cloudy to cloud-free atmosphere (Koren et al., 2007). This transition zone (the twilight zone) may influence the measured optical properties of the suspended aerosol (Radke and Hobbs, 1991; Feingold and Morley, 2003; Charlson et al., 2007) due to aerosol humidification processes (Feingold and Morley, 2003; Twohy et al., 2009), signal contribution of undetected clouds (Koren et al., 2007,

Published by Copernicus Publications on behalf of the European Geosciences Union. 
2008, 2009), and cloud 3-D radiative effects (Marshak et al., 2006; Wen et al., 2007, 2008). It was also found that this intermediating area may extend to a distance of $30 \mathrm{~km}$ from the detectable clouds (Koren et al., 2007; Twohy et al., 2009). The twilight zone properties are considered to be determined by its cloudy environment, and therefore these zones should be distinguished from the cloud-free areas. Based on this and on the current understanding of the special properties of cloud fields, Bar-Or et al. (2010) suggested to consider the cloud affected area (i.e. cloud field) by classifying the atmosphere into two: cloud fields and cloud-free, noting that the cloud class is a subset of cloud fields. Such classification requires a robust analytical definition of a cloud field that will enable the determination of its boundaries, independently on the resolution of the input cloud mask data.

Finding a robust method to define cloud field boundaries is challenging, taking into consideration the complexity of clouds and the twilight zone characteristics. Previous studies investigated mostly the statistical properties of cloud fields' spatial structure without special attention to their boundaries. The main goals of the past research were: (1) improving the inputs and the parameterizations of cloud fields in coarse resolution climate models (Davis, 1990), (2) creating synthetic (but realistic) cloud fields for numerical models usage (Venema et al., 2006), (3) estimating cloud and precipitation spatial variation while extracting the fractal and multi-fractal nature of cloud fields (Lovejoy and Mandelbrot, 1985; Cahalan and Joseph, 1989; Joseph and Cahalan, 1990; Lovejoy and Schertzer, 1990, 2006; Sengupta et al., 1990; Tessier et al., 1993), and (4) exploring the power-law size distribution of clouds and the inner properties of cloud fields (Sengupta et al., 1990; Astin and Latter, 1998; Nair et al., 1998; Koren et al., 2008).

In addition, an extensive research has been conducted in order to estimate the regularity and clustering properties of cloud fields, using morphological techniques (Weger et al., 1992, 1993; Zhu et al., 1992; Lee et al., 1994; Nair et al., 1998). In these studies, a comparison was done between the nearest neighbor cumulative distribution function (NNCDF) of cloud fields and the theoretical Poisson NNCDF in order to find the level of spatial randomness or regularity of cloud fields. It was found, for example, that for cumulus $(\mathrm{Cu})$ clouds smaller than $1 \mathrm{~km}$ in diameter, the average nearestneighbor distances equal to 3-7 cloud diameters. For larger clouds, the ratio of cloud nearest-neighbor distance to cloud diameter increases sharply with increasing cloud diameter (Zhu et al., 1992).

In this work, unlike past research, we concentrate on the boundaries of the cloud field, rather than on its inner spatial properties. For this purpose we use a morphological algorithm to analytically define cloud field boundaries, first introduced in Bar-Or et al. (2010). We provide here an extended detailed description of the algorithm. We apply this algorithm to a global cloud field coverage analysis for the first time, exposing strong climatic signature of the global circu- lation on cloud field coverage over lands. Moreover, we add global analysis of aerosol optical depth (AOD) and aerosol fine-mode fraction (FMF) as a function of the distance from the nearest cloud, based on more than 1 million pixels data.

In Sect. 2 we discuss the theoretical basis for the method. In Sect. 2.1 we describe the cloud field boundary detection algorithm in detail. In Sect. 3.1 we present global analysis of cloud field spatial and radiative properties, based on the cloud field boundary detection algorithm. In Sects. 3.2 and 3.3 we present a global statistical analysis of the mean aerosol optical depth and of the mean aerosol fine-mode fraction, as a function of the distance from the nearest cloud. We summarize in Sect. 4.

\section{Theory and method}

We define a cloud field as the area that contains both detectable clouds and the space around them within a characteristic distance from each detectable cloud. We assume that the likelihood to have an undetectable cloud (weak optical signature and/or small relative to the sensor resolution) increases as one approaches detectable clouds (Koren et al., 2007, 2008). Therefore, this likelihood is higher in a cloud field and decreases as moving away from it. Moreover, the same behavior applies for pockets of high relative humidity and extra illumination coming from the sides of clouds (Marshak et al., 2009, 2006, 2008; Varnai and Marshak, 2009; Wen et al., 2007; Zinner et al., 2008).

A robust and simple to implement cloud field masking algorithm should comply with the following requirements: (1) the algorithm should use basic input data on cloud distribution, like binary cloud mask, (2) the algorithm should be based on a spatial analysis scheme, (3) the algorithm should be applicable as long as the data resolution allows for a detailed analysis of the cloud filed properties. Hence, the resolution should be on the order or finer than a characteristic cloud size, (4) the algorithm should be valid to all cloud types.

All previous studies of cloud fields spatial structure have focused on the spatial distributions of clouds inside cloud fields, and usually considered the entire examined domain as a part of a cloud field (without any definition of the cloud field boundaries). Therefore, this work focuses on developing a robust and easy to implement cloud field boundary detection algorithm.

Few existing spatial analysis methods were considered for this research. For example, one proposed a method using joint statistics and k-nearest-neighbor (k-NN) techniques (Sankaranarayanan et al., 2007) over binary cloud mask data, in order to isolate the largest clusters in the examined domain, and then define the cloud field boundaries as the cluster edges. This method lacks the ability to treat isolated small cloud fields, and ignores these important fields. In addition, this method focuses on clouds only and does not include the 
important twilight zone in the cloud field area. Other methods that do mark a characteristic distance from the cloud field center (Koren et al., 2009a), lack the ability to mark reasonable borders when the cloud field is not rounded shape (most cloud fields are not rounded).

A cloud field masking algorithm requires a flexible method that will follow any cloud field shape. Here, that requirement is defined as "locality", i.e. the algorithm should be sensitive to scales which are higher than the scale of the whole cloud field in order to mask fields with relatively complex shape. Therefore, the preferred metric should rely on local properties of the cloud distribution. The best metric that meets this requirement was found to be the distribution of the distance-from-nearest-cloud (Koren et al., 2007), where each element represents the Euclidian distance of the center of the pixel to the nearest cloud. Such metric is local by definition and it considers nothing but the distances in the vicinity of each cloud.

Euclidian distance methods are being used in a wide range of spatial analysis applications, such as linear and edge detection of objects in digital images (Rosin, 2009), fractal dimension analysis of 2-D objects (Adler and Hancock, 1994), and recently - for cloud spatial and radiative properties (Koren et al., 2007; Marshak et al., 2008; Bar-Or et al., 2010). The last is gaining an increased interest, and a dataset of the distance-from-the-nearest-cloud is planned to be added to the MODIS atmosphere level 2 products. Deeper mathematical discussion and further synthetic examples of the distance field distribution can be found in Ripley (1981). Here, the distribution of the Euclidian distance from the nearest cloud is being used for distinguishing the inner cloud field area from the surrounding cloud-free area (see Fig. 1b). Examining the whole domain (including the cloud-free area), the distance distribution shows two different regimes: (1) the intra cloud-field regime, characterized by the distance distribution of clouds inside the field (describing the cloud spatial distribution, Fig. 1c), and (2) the extra-field regime, which asymptotically approximate distance distribution of a single giant cloud (see in Fig. 1d).

While the distance distribution inside the field has a maximum point, representing the most common distance from a cloud inside the field, following by a decrease of larger distances (Fig. 1e), the distance distribution outside the fields is monotonically increasing (with a slope that asymptotically goes to $2 \pi$, away from the cloud field as the smoothed perimeter approximate a circle, see Fig. 1d).

Having a local maximum in the intra cloud field distribution and monotonic increase out of the cloud field defines a minimum in the transition between the distributions.

The distance value corresponding to the local minimum is defined here as the field distance parameter $\left(R_{0}\right)$, and it represents the largest distance-from-the-nearest-cloud that is still considered to be part of the cloud field, based on the assumption that each cloud field has a limited distance between neighboring clouds, and therefore limited intra-field distance-from-the-nearest-cloud. The contour defined by $R_{0}$ marks the cloud field boundaries, distinguishing the cloud field from the surrounding cloud-free area, and demonstrating the ability of $R_{0}$ to define cloud field boundaries without making any prior assumption about their geometrical shape. Figure 1 shows a distance map and a distribution of the distance from the nearest cloud for a synthetic cloud field. It clearly shows the different distribution properties inside and outside of the cloud field. A true data example for the extraction of the field distance parameter is presented in Fig. 2.

\subsection{The algorithm}

To implement the described method on observational data, additional data analysis is required. The complete cloud field bounding algorithm for the MODIS data is described below:

- Step 1 -data projection on an equal-area matrix The large footprint of the MODIS instrument results in slight geometrical distortion. The first step of the algorithm is a projection of all product granules (data blocks) on an equal-area matrix, in order to avoid any errors due to geometrical differences. The input data for this work is the MODIS cloud mask product (Ackerman et al., 1998; Platnick et al., 2003), as all pixels that are determined to be confidently cloudy according to this product were considered to be clouds. The projection to $1 \mathrm{~km}^{2}$ equal-area cloud mask and to $1 \mathrm{~km}^{2}$ equal-area ocean/land masks are done using MODIS Geolocation product. The Euclidian distance of each pixel in the MODIS product to a central point is calculated, based on its exact coordinates, enabling the interpolation of the required data on a new equal-area grid of $1 \mathrm{~km}$ pixels. The borders of the cloud mask interpolations were rounded.

\section{- Step 2 - distance map extraction}

The distance from the nearest cloud is calculated, based on the $1 \mathrm{~km} /$ pixel equal-area cloud mask. Additional domain size correction is done by cutting out from the data all pixels whose calculated distance from the nearest cloud is larger than their distance to the granule edge.

- Step 3 - calculating the distance distribution

The distance cumulative distribution $A(r)$ is calculated for varying distance parameter values $(r) . A(r)$ is the total area that is closer than $r$ from any cloud in the observed domain. Then, the distance distribution is calculated as the derivative. This procedure is equivalent to computing the distance distribution as the histogram of the distance map, but is technically faster and more sensitive to the minimum location.

- Step 4-noise corrections for the distance distribution The generated distance distribution $\frac{d}{d r}(A(r))$ may suffer from high noise levels, mostly for low $r$ values when 

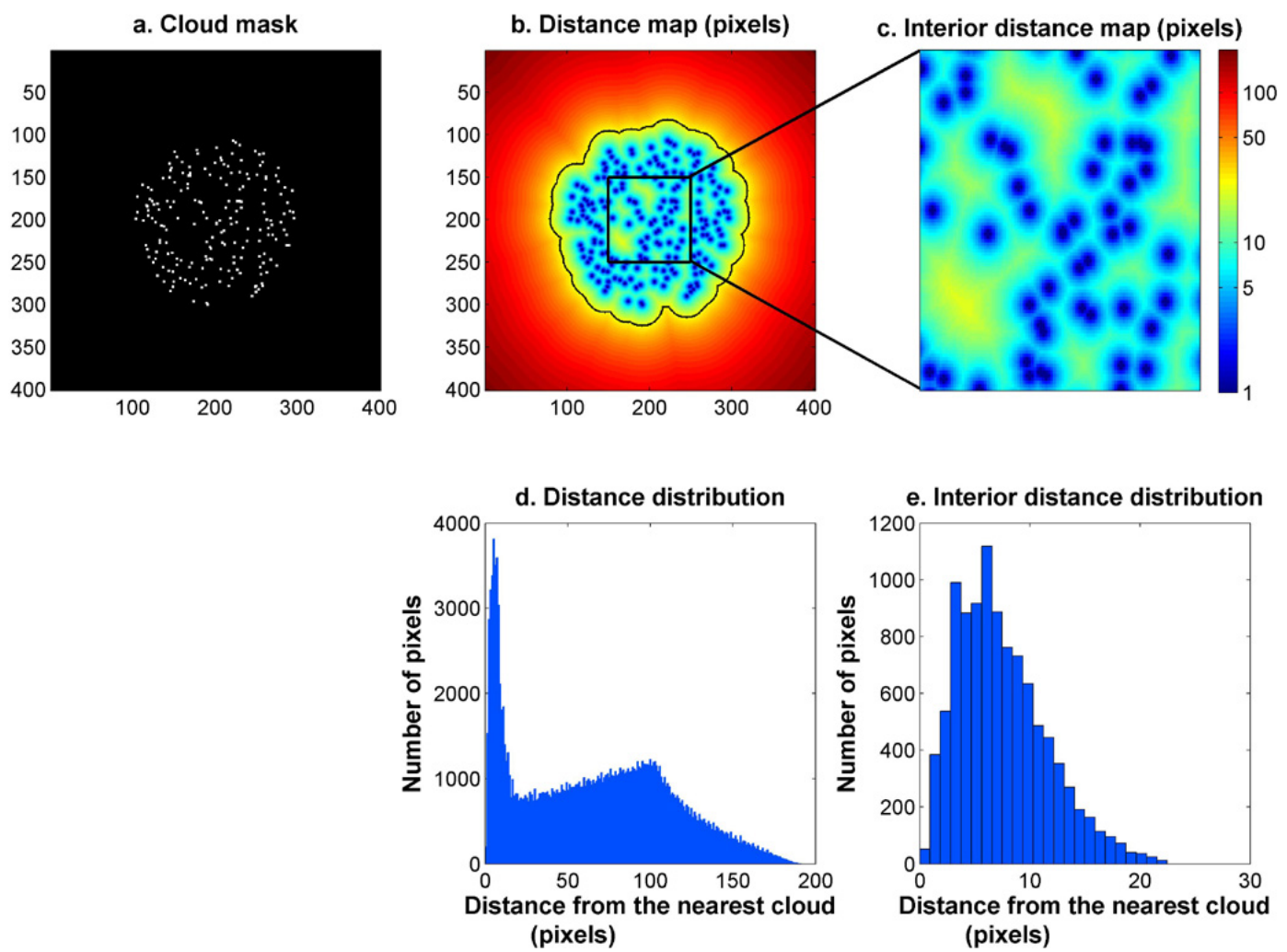

Fig. 1. A synthetic cloud field, containing pixel size randomly distributed clouds (a), the field's distance map (b, logarithmic scale), and a zoom on its interior distance map (c, logarithmic scale). The different distance distributions of the whole synthetic field and of its interior (d and $\mathbf{e}$, respectively) enable the detection of the transition point between the intra field distances and the complete field distances. In this case, the transition point appears at $r=22$ pixels, and its derived cloud field boundary is marked (panel $\mathbf{b}$, black line). The decrease of the distance distribution in panel $\mathbf{d}$ (from distances of 100 pixels and more) is due to the restricted domain size where larger distances are needed. Note that while Fig. 1c represents a zoomed image of Fig. 1b, the corresponding Fig. 1e represents only an approximated scaled zoom of the interior distances in Fig. 1d (up to $\sim 22$ pixels), neglecting all distances that describe cloud-free areas.

the distances calculated for integer number of pixels may create discontinuities in the distribution. In this step, a Gaussian filter is applied on the distance distribution in order to filter out high frequency variations, and to enable the calculation of field distance parameter $R_{0}$. Figure 2 demonstrates the distance cumulative function $A(r)$, the distance distribution $\frac{d}{d r}(A(r))$, and the smoothed distance distribution function.

- Step 5 - extracting the field distance parameter $R_{0}$ The local minimum of the smoothed distance distribution function is used to determine the field distance parameter $R_{0}$, as demonstrated in Fig. 2.

- Step 6 - calculating the cloud field boundaries and coverage

After determining $R_{0}$, the Cloud Field Fraction (CFF) is calculated. The cloud field fraction is a unitless normalized ratio that represents the portion of cloud field covered area in the whole examined domain and defined as: $\mathrm{CFF}=\frac{A\left(R_{0}\right)}{A_{\mathrm{D}}}$, where $A_{\mathrm{D}}$ is the domain's area. The $\mathrm{CFF}$ is analogue to the cloud fraction measure. $\mathrm{CFF}=0$ represents an absolute cloud-free domain and $\mathrm{CFF}=1 \mathrm{a}$ domain that contains only cloud field area. Given that the domain's cloud fraction is $\frac{A(r=0)}{A_{\mathrm{D}}}$, the domain's CFF is always equal or larger than the domain's cloud fraction. The boundaries of the cloud field are represented by the contour $r=R_{0}$ where all the pixels whose distance from the nearest cloud is smaller than $R_{0}$ are part of the field.

\subsection{Sensitivity and limitations}

The proposed algorithm was examined by an extensive set of sensitivity tests, verifying that the algorithm is not sensitive to the resolution of the input cloud mask data or to the clouds' spatial distribution. The tests were conducted on both synthetic and realistic (observed by MODIS) cloud fields. For these tests, the resolution of each cloud mask data was reduced by a simple averaging of pixels, and setting all pixels averaged higher than 0.5 as cloudy, and all pixels averaged lower than 0.5 to clear. Pixels whose average was exactly 0.5 , were randomly set as cloudy or clear (with the probability of 


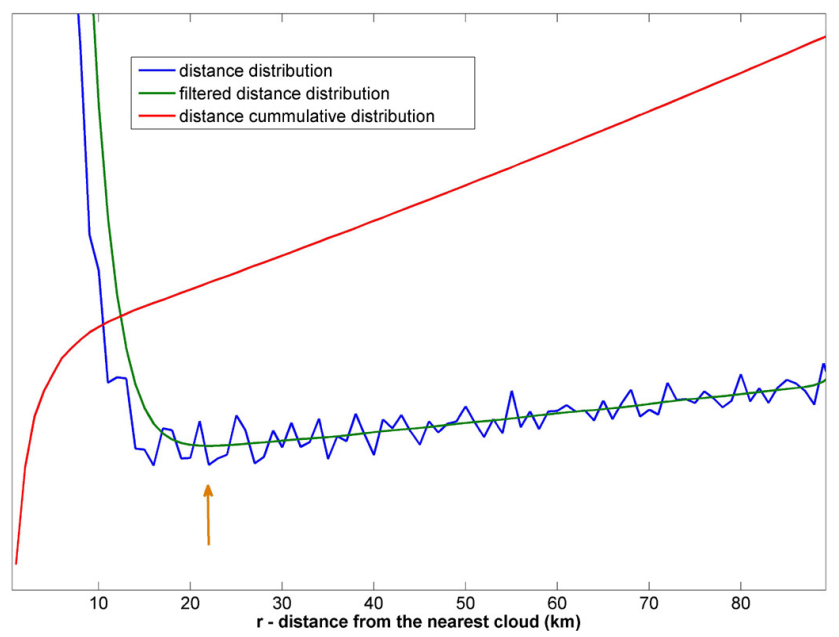

Fig. 2. Analysis of an observed cloud field, including the distance distribution $d A(r) / d r$ (blue line, normalized), the filtered distance distribution function (green line, normalized), the distance cumulative distribution $A(r)$ (red line, normalized), and the transition point, identified by the minimum of the filtered distance distribution function, and defining the field distance parameter (marked with an orange arrow).

$1 / 2$ ), keeping the reduced resolution data statistically unbiased, and verifying that the cloud fraction is constant. After reducing the data resolution (i.e. increasing the data pixel size), the algorithm was used for calculating the field distance parameter. The algorithm was found to be stable for varying data resolutions, provided that the data pixel size is smaller than the characteristic length scale of the examined cloud field. Figure 3 demonstrates the described resolution sensitivity test for a MODIS observed cloud field (the same as was analyzed in Fig. 2). In this case, the field distance parameters calculated by the algorithm are in the range of $17.5-22.0 \mathrm{~km}$, for pixel size smaller than $7 \mathrm{~km}$. The cloud fraction is stable in the range of $62.6 \%-64.6 \%$, as expected by a resolution reduction of a binary cloud mask.

Using cloud mask as its only input data, the algorithm is sensitive to the technique and to the quality of the chosen cloud mask product. One must consider this limitation, although there is no satisfying alternative that provides better cloud spatial data.

For the sake of completeness, the theoretical case of a cloud field that contains only one circular cloud is also considered. In this case the distance distribution function has only one minimum at $r=0$, and therefore the calculated field distance parameter is zero. These scenarios are easily identified by the algorithm and gain field distance parameters that are defined by the mean field distance parameters of the neighboring cloud fields. However, the probability to find such cloud fields in realistic datasets is negligible.

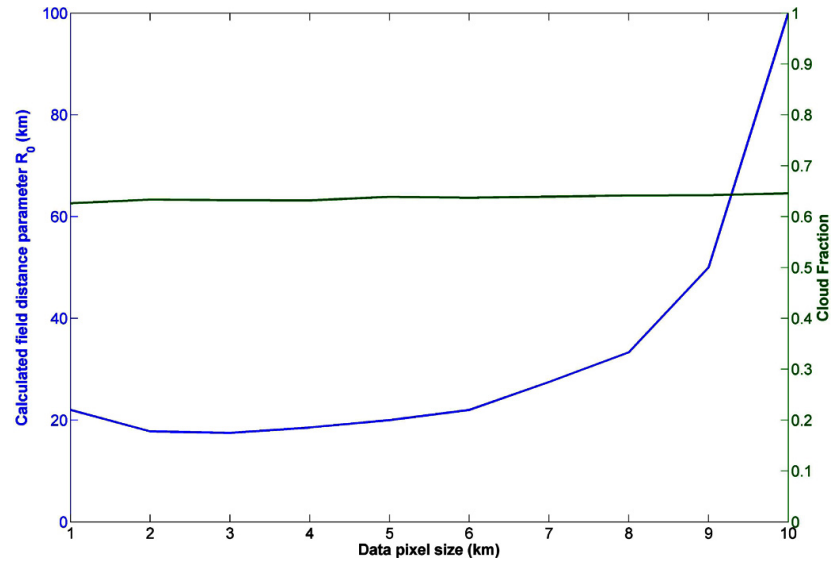

Fig. 3. The calculated field distance parameter $R_{0}$ (blue line) and the relative cloud fraction (green line), for varying resolution of MODIS data.

\section{Analysis and results}

The data analyzed in this section are MODIS-Terra atmosphere level 2 products (Platnick et al., 2003; Remer et al., 2005; Levy et al., 2007), for one day at 28 July 2008. The presented results are based on analysis of 66 granules (dataset blocks), containing day-light information (for this specific day) between latitudes $50^{\circ} \mathrm{S}-50^{\circ} \mathrm{N}$. The cloud mask input data are based on the MODIS cloud mask product (Ackerman et al., 1998; Platnick et al., 2003), the aerosol properties data are based on the MODIS aerosol product (Remer et al., 2005; Levy et al., 2007), and both sea/land mask and geo-location data are based on MODIS Geolocation product.

\subsection{The global cloud field fraction}

The global CFF $\left(50^{\circ} \mathrm{S}\right.$ to $\left.50^{\circ} \mathrm{N}\right)$ is estimated over land and ocean. In order to find whether there is any dependence of the field distance parameter on the cloud type, several cloud fields in each granule are manually classified. This classification is done using the MODIS provided true-color images, based on the familiar spatial morphology of the clouds type. The classification included four cloud types: Stratocumulus $(\mathrm{Sc})$, shallow Cumulus $(\mathrm{Cu})$, Cirrus $(\mathrm{Ci})$, and deep convective (DC).

The field distance parameter of each of the selected 170 cloud fields is calculated using the described algorithm; which treats all cloud types in a similar way.

Past research of the twilight zone showed clear exponential decay of several atmospheric properties when increasing the distance from the nearest cloud. The exponential decaying features include the reflectance, the aerosol optical depth (Marshak et al., 2006; Koren et al., 2007; Chiu et al., 2009), and the relative humidity (Koren et al., 2009b; Twohy et al., 2009). Koren et al. (2007) showed that the twilight e-fold 
Table 1. Granule cloud fraction (CF), cloud field fraction (CFF), and field distance parameters $\left(R_{0}\right)$ as calculated globally for 28 July 2008. The cloud fields are classified to: $\mathrm{Ci}, \mathrm{Sc}, \mathrm{Cu}$, and Deep Convective (DC). It refers both to fields and granules, respectively.

\begin{tabular}{lrrr}
\hline & Mean & $\begin{array}{r}\text { Standard } \\
\text { deviation }\end{array}$ & $\begin{array}{r}\text { Number } \\
\text { of Samples }\end{array}$ \\
\hline Granule Cloud Fraction & $51 \%$ & $23 \%$ & 66 \\
Granule CFF (Calculated $\left.R_{0}\right)$ & $88 \%$ & & 66 \\
Granule CFF $\left(R_{0}=10 \mathrm{~km}\right)$ & $80 \%$ & $23 \%$ & 66 \\
$R_{0}-\mathrm{All}$ & $29 \mathrm{~km}$ & $9 \mathrm{~km}$ & 170 \\
$R_{0}-\mathrm{Ci}$ & $30 \mathrm{~km}$ & $9 \mathrm{~km}$ & 38 \\
$R_{0}-\mathrm{Sc}$ & $25 \mathrm{~km}$ & $8 \mathrm{~km}$ & 21 \\
$R_{0}-\mathrm{Cu}$ & $29 \mathrm{~km}$ & $9 \mathrm{~km}$ & 80 \\
$R_{0}-\mathrm{DC}$ & $31 \mathrm{~km}$ & $8 \mathrm{~km}$ & 31 \\
\hline
\end{tabular}

distance from the nearest cloud is $\sim 10 \mathrm{~km}$. Based on that, we estimate the global CFF also by using a constant distance parameter $\left(R_{0}=10 \mathrm{~km}\right)$.

The results of the global CFF and of the field distance parameters of the examined cloud fields during 28 July 2008 are presented in Table 1.

The results show a global average field distance parameter of $29 \pm 1 \mathrm{~km}$, which agrees with the results of Bar-Or et al. (2010), that found a monthly averaged field distance parameter of $30 \mathrm{~km}$ over the Atlantic Ocean during July 2008 (the error is calculated as a standard mean error). The results also agree with Koren et al. (2007), that found a reflectance signal effect up to $30 \mathrm{~km}$ from the nearest cloud edges. The comparison between the field distance parameters of different cloud field types shows that Sc fields have the smallest distance parameter, probably due to their sharp transition to cloud-free atmosphere at their edges. The calculated field distance parameter for Cirrus cloud fields is very close to the mean value of all cloud type, possibly because Cirrus field may be located above a wider field of different cloud type. Such setting would lead the algorithm to be sensitive to the clouds that appear on the border of the fields. All calculated field distance parameter values are in the same order of magnitude of $29 \pm 9 \mathrm{~km}$, with the extreme results being 17 and $39 \mathrm{~km}$.

The global cloud field fraction for 28 July 2008 is $88 \%$, calculated using the algorithm generated field distance parameter $R_{0}$, and $81 \%$ when using the constant $R_{0}=10 \mathrm{~km}$ for the whole dataset. These results are in line with Twohy et al. (2009) that found that only $8 \%$ of the detected cloud-free area above oceans is located in a distance larger than $20 \mathrm{~km}$ from the nearest detected cloud. This suggests that the CFF over oceans, using a constant $R_{0}$ of $20 \mathrm{~km}$, is approximately $92 \%$. Koren et al. (2007) showed that an addition of $30 \mathrm{~km}$ belt around all clouds when the global cloud fraction is 51\%, will cover $81 \%$ of Earth's surface. It means a global CFF of approximately $81 \%$ when using a constant $R_{0}$ of $30 \mathrm{~km}$.
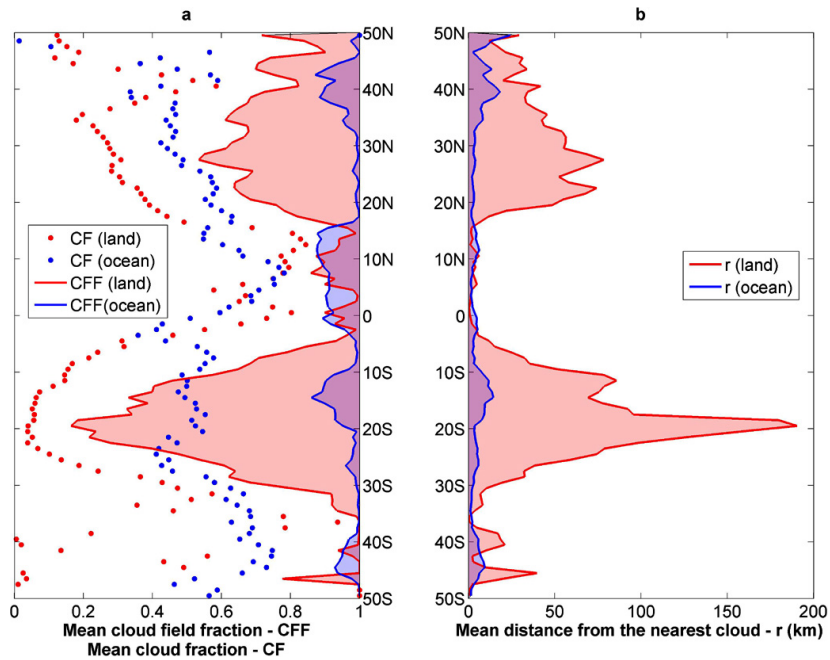

Fig. 4. Latitudinal mean cloud fraction (left panel, dots), cloud field fraction (left panel, lines) and distance from the nearest cloud (right panel), above land (red) and ocean (blue), based on MODIS Terra observations for 28 July 2008.

Next, the calculated global CFF and the distance-fromthe-nearest-cloud above land and above ocean were separated and compared for different latitudes (with a latitudinal resolution of $1^{\circ}$ ). The calculated latitudinal mean CFF, and the latitudinal mean distance-from-the-nearest-cloud are presented in Fig. 4.

The results show a clear difference between cloud fields above oceans and lands. While the CFF above oceans is $80 \%$ or higher in all latitudes, the CFF above land carries a strong signature of the global atmospheric circulation. The CFF above land significantly decreases in the Hadley subsidence (desert belt) latitudes $\left(10^{\circ} \mathrm{S}-25^{\circ} \mathrm{S}\right.$ and $\left.20^{\circ} \mathrm{N}-35^{\circ} \mathrm{N}\right)$. The northward shift of the Hadley cells is expected considering the date of observed data, during the boreal summer (28 July 2008). Moreover, a closer examination of the differences between the $\mathrm{CF}$ and the $\mathrm{CFF}$ curves shows that while the CFF over land has a similar trend to the CF, the CFF over oceans is uncorrelated to its corresponding $\mathrm{CF}$; this indicates that the spatial cloud field structures over land significantly differ from the structures over oceans.

In spite of the low latitudinal variance of the distancefrom-the-nearest-cloud parameter, over oceans, the mean latitudinal CFF reveals a clear difference between the Hadley subsidence latitudes $\left(10^{\circ} \mathrm{S}-25^{\circ} \mathrm{S}\right.$ and $\left.20^{\circ} \mathrm{N}-35^{\circ} \mathrm{N}\right)$ and the Inter Tropical Convergence Zone (ITCZ, $\left.5^{\circ} \mathrm{S}-15^{\circ} \mathrm{N}\right)$. This transition is probably a result of the difference between the spatial properties of the marine cloud fields over the ITCZ (mostly deep convective), and the cloud fields in the subsidence zone latitudes (mostly shallow clouds). The ITCZ's distinguished marine CFF behavior demonstrates the ability of the algorithm to indentify different cloud fields by their distance distribution, in spite of their similar mean distancefrom-the-nearest-cloud values. 


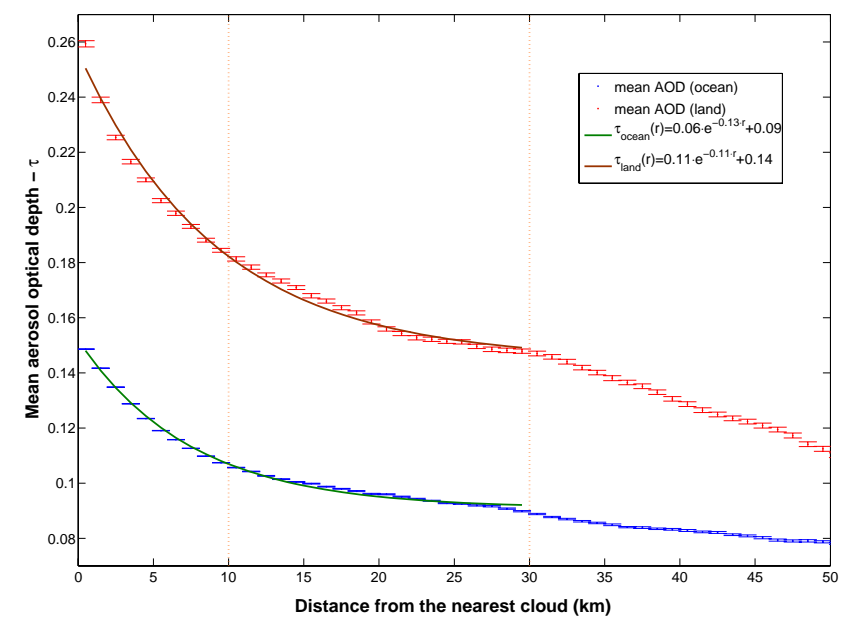

Fig. 5. Global mean aerosol optical depth (AOD) as a function of the distance from the nearest cloud, retrieved above land (red dots) and above ocean (blue dots), and the matching exponential fitting functions (purple and green lines, respectively), for distances of 0 $30 \mathrm{~km}$ from the nearest cloud. The error bars represent the standard mean error. All data are based on MODIS Terra observation for 28 July 2008. The constant distance lines highlight the values for $R_{0}=10 \mathrm{~km}$ and for the calculated global characteristic value $\left(R_{0}=\right.$ $30 \mathrm{~km}$, see Sect. 3.1).

Furthermore, these results indicate that while the likelihood to sample a total cloud-free (away from cloud fields) pixel above oceans is $\sim 12 \%$, the likelihood to sample such a pixel above land varies between $\sim 10 \%$ in the ITCZ latitudes, and $\sim 80 \%$ in the central latitudes of the Southern subsidence zone (around $20^{\circ} \mathrm{S}$, in the observed day).

\subsection{Aerosol optical depth and cloud fields}

The aerosol properties' retrievals in the vicinity of clouds are affected by aerosol humidification processes (Feingold and Morley, 2003; Twohy et al., 2009), by signal contribution of undetected clouds (Koren et al.,2007, 2008, 2009), and by cloud 3-D radiative effects (Marshak et al., 2006; Wen et al., 2007).

Here, a daily global dataset is used to show these effects, separately above land and ocean. Figure 5 presents the mean AOD as a function of the distance-from-the-nearest-cloud, showing a clear exponential decay. The higher mean AOD values above land (compared to the oceans) are an expected result because of the higher aerosol concentrations observed above land.

Unlike the analysis presented in Bar-Or et al. (2010), where Cirrus cloud fields were excluded from the input data, in this analysis all cloud types are included. The optical contribution of the twilight zone around Cirrus clouds is considered in this global analysis because undetectable Cirrus clouds often appear in the vicinity of detectable clouds and affect the aerosol optical retrieval.
The mean AOD as a function of the distance-from-thenearest-cloud is monotonically decreasing. An interesting finding is the clear change in the decrease rate, around $30 \mathrm{~km}$ distance from the nearest cloud. Given the large size of the dataset (more than 1 million pixels), this sharp rate change may point to a characteristic influence scale of cloud fields, supporting the findings presented in Sect. 3.1, and previous works (Koren et al., 2007; Remer et al., 2008; Twohy et al., 2009). The exponential decay found here agrees with previous findings and the coefficients presented here agree with the values calculated by Bar-Or et al. (2010). The global mean AOD values found here are also supported by the MODIS-Terra long-term global analysis of Remer et al. (2008) that found a global mean AOD of 0.19 above land, and of 0.13 above ocean.

These results are strengthening the need to distinguish between measurements of aerosols inside and outside cloud fields, because of the significant difference in both actual aerosol properties and the measured aerosol optical characteristics near detectable clouds.

\subsection{Aerosol fine-mode fraction and cloud fields}

The retrieved measures for aerosol size distribution are also affected by nearby clouds, mainly by the contribution of undetected clouds and high humidity areas in the vicinity of clouds, causing swelling of aerosols (Pahlow et al., 2006).

Here we test the sensitivity of the MODIS aerosol fine mode fraction (FMF) to the distance from the nearest cloud. The data for this analysis is selected to be only above oceans due to the high uncertainties in the aerosol fine-mode fraction product retrieved above land (Remer et al., 2005). Figure 6 presents the mean FMF values retrieved above ocean as a function of the distance-from-the-nearest-cloud. The results clearly show two regimes in the graph. The first, in the range of distances between $0 \sim 33 \mathrm{~km}$ from the nearest cloud, that shows an exponential increase of the FMF with the distance from the nearest cloud, varying from $37.1 \%$ at $0-1 \mathrm{~km}$ to $53.4 \%, 32-33 \mathrm{~km}$ from the nearest cloud. The calculated exponential fit in this regime saturates at FMF of $\sim 54 \%$. The second regime in the graph is for distances that are larger than $\sim 33 \mathrm{~km}$ that shows a slow decrease of the FMF when increasing the distance from the nearest cloud (in the range of $53.4 \%-51.7 \%$ ).

The results of the first regime in the graph can be explained by the theoretical superposition of three effects. The first is the aerosol swelling process that produces sharp exponential decay in the aerosol size, as the distance to the nearest cloud grows (Pahlow et al., 2006). The second is the effect of undetectable clouds that "increase" the aerosol apparent size, and the third is the cloud 3-D radiative effect (Wen et al., 2007), which may enhance the apparent aerosol optical depth near clouds. 


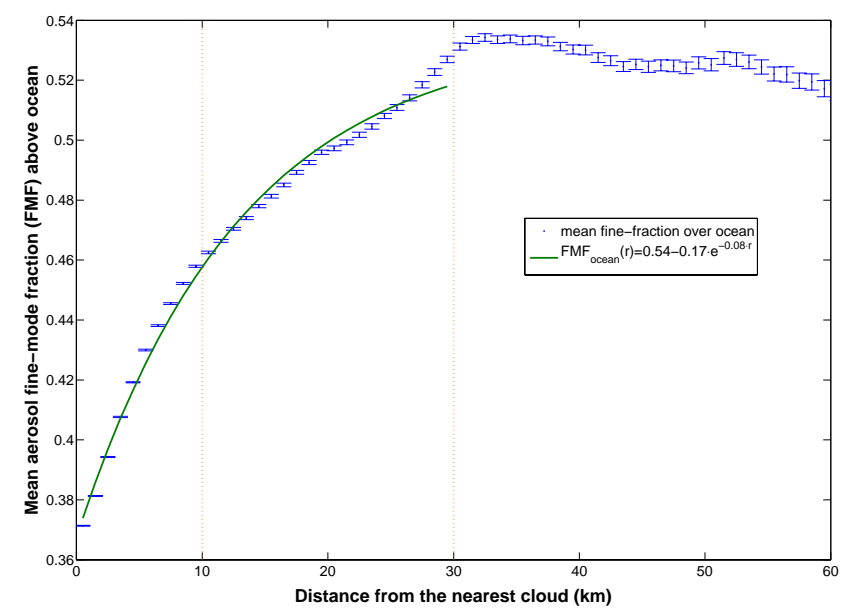

Fig. 6. Global mean aerosol fine-mode fraction (FMF) as a function of the distance from the nearest cloud, retrieved above ocean (blue dots), and its exponential fitting function (green line), for distances of $0-30 \mathrm{~km}$ from the nearest cloud. The error bars represent the standard mean error. All data are based on MODIS Terra observation for 28 July 2008. Similarly to Fig. 5, the constant distance lines highlight the values for $R_{0}=10 \mathrm{~km}$ and for the calculated global characteristic value.

\section{Summary}

This study introduces estimations of the global cloud field coverage with special examination of its latitudinal dependence. It presents analysis of the retrieved aerosol properties, with respect to its location within cloud fields. It shows that the previously suggested estimate of the $30 \mathrm{~km}$ scale (Koren et al., 2007), as a typical distance that one should take from the nearest detectable cloud in order to be away from the twilight zone (i.e., outside of a cloud field), is reproducible in many types of unrelated analyses. This suggests that the $30 \mathrm{~km}$ scale is a natural entity of cloud fields.

The algorithm for defining cloud field boundaries, presented here in detail, is shown to be robust and simple for implementation. This algorithm uses a binary cloud mask as the input for the clouds boundaries, and estimates the characteristic distance $R_{0}$ from the nearest cloud: a distance that separates the cloud affected atmosphere from the cloud-free atmosphere, outside of cloud fields. The global cloud field fraction (CFF) is estimated, using this algorithm and MODIS cloud mask ( $50^{\circ} \mathrm{S}$ to $50^{\circ} \mathrm{N}, 170$ cloud fields in 66 granules).

The estimated global CFF for 28 July 2008 is $88 \%$ when calculated by the conservative cloud field bounding algorithm, and $81 \%$ when using fixed $R_{0}=10 \mathrm{~km}$, assuming that more than $2 / 3$ of the cloud effect area is concentrated in the closest $10 \mathrm{~km}$ from the detectable cloud (based on the exponential decay of AOD and reflectance).

Latitudinal analysis of the global CFF shows that the mean oceanic CFF is higher than $\sim 80 \%$ in all latitudes, while the mean $\mathrm{CFF}$ above land carries a strong signature of the global atmosphere circulation, reducing the mean CFF over the desert belts, where the Hadley cell subsidizes, to extremely low values of $\sim 20 \%$. These findings suggest that the average likelihood to sample a total cloud-free $1 \mathrm{~km}$ pixel above oceans is lower than $20 \%$ in all latitudes, and may be $\sim 80 \%$ above land in the Southern Hadley subsidence zone.

Global analysis of the influence of cloud fields on the aerosol optical depth (AOD) retrieval and on the aerosol finemode fraction (FMF) is examined. An exponential decay of the mean AOD as a function of the distance-from-thenearest-cloud is found both above land and above ocean. This finding reveals a significantly higher mean AOD inside cloud fields, strengthening the need to distinguish between measurements of aerosols inside and outside cloud fields.

An additional global analysis of the aerosol fine-mode fraction (FMF) found an exponential increase of the FMF as a function of the distance-from-the-nearest-cloud, supporting the presence of humidified aerosols and undetectable clouds near detectable clouds. However, this exponential increase dominates only distances in range of $0 \sim 33 \mathrm{~km}$ from the nearest cloud, suggesting a physical transition zone located $\sim 30 \mathrm{~km}$ between cloud field inner part and the free atmosphere.

All above results raise the need to treat cloud fields as a major and important entity in the atmosphere. It is suggested here that cloud fields and cloud-free atmosphere should be investigated separately, considering both detectable clouds and their close environment as a cloud field. The presented algorithm is shown to be robust and useful for such separation, supporting future aerosol-cloud-climate studies.

Acknowledgements. This article is dedicated to the memory of Yoram J. Kaufman, a dear friend and a brilliant scientist. This work was supported by the Sussman Family Center of Environmental Research. RB is supported by the Rieger Foundation JNF Fellowship 2009/2010.

Edited by: B. Mayer

\section{References}

Ackerman, S. A., Strabala, K. I., Menzel, W. P., Frey, R. A., Moeller, C. C., and Gumley, L. E.: Discriminating clear sky from clouds with MODIS, J.Geophys. Res.-Atmos., 103, 3214132157, 1998.

Adler, J. and Hancock, D.: Advantages of using a distance transform function in the measurement of fractal dimensions by the dilation method, Powder Technol., 78, 191-196, 1994.

Astin, I. and Latter, B. G.: A case for exponential cloud fields?, J. Appl. Meteorol., 37, 1375-1382, 1998.

Bar-Or, R. Z., Koren, I., and Altaratz, O.: Estimating cloud field coverage using morphological analysis, Environ. Res. Lett., 5, 014022, doi:10.1088/1748-9326/5/1/014022, 2010.

Cahalan, R. F. and Joseph, J. H.: Fractal statistics of cloud fields, Mon. Weather Rev., 117, 261-272, 1989.

Charlson, R. J., Ackerman, A. S., Bender, F. A. M., Anderson, T. L., and Liu, Z:: On the climate forcing consequences of the albedo 
continuum between cloudy and clear air, Tellus B, 59, 715-727, doi:10.1111/j.1600-0889.2007.00297.x, 2007.

Chiu, J. C., Marshak, A., Knyazikhin, Y., Pilewski, P., and Wiscombe, W. J.: Physical interpretation of the spectral radiative signature in the transition zone between cloud-free and cloudy regions, Atmos. Chem. Phys., 9, 1419-1430, doi:10.5194/acp-91419-2009, 2009.

Davis, T. J.: A simple parameterization scheme for joint statistics of cloud field morphology and physical parameters, J. Appl. Meteorol., 29, 776-782, 1990.

Dybbroe, A., Karlsson, K. G., and Thoss, A.: NWCSAF AVHRR cloud detection and analysis using dynamic thresholds and radiative transfer modeling. Part I: Algorithm description, J. Appl. Meteorol., 44, 39-54, 2005.

Feingold, G. and Morley, B.: Aerosol hygroscopic properties as measured by lidar and comparison with in situ measurements, $\mathrm{J}$. Geophys. Res.-Atmos., 108, 4327, doi:10.1029/2002jd002842, 2003.

Joseph, J. H. and Cahalan, R. F.: Nearest neighbor spacing of fair weather cumulus clouds, J. Appl. Meteorol., 29, 793-805, 1990.

Kaufman, Y. J., Tanre, D., and Boucher, O.: A satellite view of aerosols in the climate system, Nature, 419, 215-223, doi:10.1038/nature01091, 2002.

King, M. D., Menzel, W. P., Kaufman, Y. J., Tanre, D., Gao, B. C., Platnick, S., Ackerman, S. A., Remer, L. A., Pincus, R., and Hubanks, P. A.: Cloud and aerosol properties, precipitable water, and profiles of temperature and water vapor from MODIS, IEEE T. Geosci. Remote., 41, 442-458, doi:10.1109/tgrs.2002.808226, 2003.

Koren, I., Remer, L. A., Kaufman, Y. J., Rudich, Y., and Martins, J. V.: On the twilight zone between clouds and aerosols, Geophys. Res. Lett., 34, L08805, doi:10.1029/2007g1029253, 2007.

Koren, I., Oreopoulos, L., Feingold, G., Remer, L. A., and Altaratz, O.: How small is a small cloud?, Atmos. Chem. Phys., 8, 3855-3864, doi:10.5194/acp-8-3855-2008, 2008.

Koren, I., Feingold, G., Jiang, H. L., and Altaratz, O.: Aerosol effects on the inter-cloud region of a small cumulus cloud field, Geophys. Res. Lett., 36, L14805, doi:10.1029/2009g1037424, 2009.

Lee, J., Chou, J., Weger, R. C., and Welch, R. M.: Clustering, randomness, and regularity in-cloud fields.4. Stratocumulus cloud fields, J. Geophys. Res.-Atmos., 99, 14461-14480, 1994.

Levy, R. C., Remer, L. A., Mattoo, S., Vermote, E. F., and Kaufman, Y. J.: Second-generation operational algorithm: Retrieval of aerosol properties over land from inversion of Moderate Resolution Imaging Spectroradiometer spectral reflectance, J. Geophys. Res.-Atmos., 112, D13211, doi:10.1029/2006jd007811, 2007.

Lovejoy, S. and Mandelbrot, B. B.: Fractal properties of rain, and a fractal model, Tellus A, 37, 209-232, 1985.

Lovejoy, S. and Schertzer, D.: Fractals, raindrops and resolution dependence of rain measurements, J. Appl. Meteorol., 29, 11671170, 1990.

Lovejoy, S. and Schertzer, D.: Multifractals, cloud radiances and rain, J. Hydrol., 322, 59-88, doi:10.1016/j.jhydro1.2005.02.042, 2006

Luo, Y., Trishchenko, A. P., and Khlopenkov, K. V.: Developing clear-sky, cloud and cloud shadow mask for producing clear-sky composites at 250-meter spatial resolution for the seven MODIS land bands over Canada and North America, Remote Sens. Env- iron., 112, 4167-4185, doi:10.1016/j.rse.2008.06.010, 2008.

Marshak, A., Platnick, S., Varnai, T., Wen, G. Y., and Cahalan, R. F.: Impact of three-dimensional radiative effects on satellite retrievals of cloud droplet sizes, J. Geophys. Res.-Atmos., 111, D09207, doi:10.1029/2005jd006686, 2006.

Marshak, A., Wen, G., Coakley, J. A., Remer, L. A., Loeb, N. G., and Cahalan, R. F.: A simple model for the cloud adjacency effect and the apparent bluing of aerosols near clouds, J. Geophys. Res.-Atmos., 113, D14S17, doi:10.1029/2007jd009196, 2008.

Marshak, A., Knyazikhin, Y., Chiu, J. C., and Wiscombe, W. J.: Spectral invariant behavior of zenith radiance around cloud edges observed by ARM SWS, Geophys. Res. Lett., 36, L16802, doi:10.1029/2009g1039366, 2009.

Nair, U. S., Weger, R. C., Kuo, K. S., and Welch, R. M.: Clustering, randomness, and regularity in cloud fields -5 . The nature of regular cumulus cloud fields, J. Geophys. Res.-Atmos., 103, 11363-11380, 1998.

Pahlow, M., Feingold, G., Jefferson, A., Andrews, E., Ogren, J. A., Wang, J., Lee, Y. N., Ferrare, R. A., and Turner, D. D.: Comparison between lidar and nephelometer measurements of aerosol hygroscopicity at the Southern Great Plains Atmospheric Radiation Measurement site, J. Geophys. Res.-Atmos., 111(8), D05s15, doi:10.1029/2004jd005646, 2006.

Platnick, S., King, M. D., Ackerman, S. A., Menzel, W. P., Baum, B. A., Riedi, J. C., and Frey, R. A.: The MODIS cloud products: Algorithms and examples from Terra, IEEE T. Geosci. Remote., 41, 459-473, doi:10.1109/tgrs.2002.808301, 2003.

Radke, L. F. and Hobbs, P. V.: Humidity and particle fields around some small cumulus clouds, J. Atmos. Sci., 48, 1190-1193, 1991.

Remer, L. A., Kaufman, Y. J., Tanre, D., Mattoo, S., Chu, D. A., Martins, J. V., Li, R. R., Ichoku, C., Levy, R. C., Kleidman, R. G., Eck, T. F., Vermote, E., and Holben, B. N.: The MODIS aerosol algorithm, products, and validation, J. Atmos. Sci., 62, 947-973, 2005.

Remer, L. A., Kleidman, R. G., Levy, R. C., Kaufman, Y. J., Tanre, D., Mattoo, S., Martins, J. V., Ichoku, C., Koren, I., Yu, H. B., and Holben, B. N.: Global aerosol climatology from the MODIS satellite sensors, J. Geophys. Res.-Atmos., 113(18), D14s07, doi:10.1029/2007jd009661, 2008.

Rosin, P. L.: A simple method for detecting salient regions, Pattern Recognit., 42, 2363-2371, doi:10.1016/j.patcog.2009.04.021, 2009.

Sankaranarayanan, J., Samet, H., and Varshney, A.: A fast all nearest neighbor algorithm for applications involving large point-clouds, Computers \& Graphics-Uk, 31, 157-174, doi:10.1016/j.cag.2006.11.011, 2007.

Sengupta, S. K., Welch, R. M., Navar, M. S., Berendes, T. A., and Chen, D. W.: Cumulus cloud field morphology and spatial patterns derived from high spatial-resolution landsat imagery, J. Appl. Meteorol., 29, 1245-1267, 1990.

Tessier, Y., Lovejoy, S., and Schertzer, D.: Universal multifractals theory and observations for rain and clouds, J. Appl. Meteorol., 32, 223-250, 1993.

Twohy, C. H., Coakley, J. A., and Tahnk, W. R.: Effect of changes in relative humidity on aerosol scattering near clouds, J. Geophys. Res.-Atmos., 114, D05205, doi:10.1029/2008jd010991, 2009.

Varnai, T. and Marshak, A.: MODIS observations of enhanced clear sky reflectance near clouds, Geophys. Res. Lett., 36, L06807, 
doi:10.1029/2008g1037089, 2009.

Venema, V., Meyer, S., Garcia, S. G., Kniffka, A., Simmer, C., Crewell, S., Lohnert, U., Trautmann, T., and Macke, A.: Surrogate cloud fields generated with the iterative amplitude adapted Fourier transform algorithm, Tellus A, 58, 104-120, 2006.

Weger, R. C., Lee, J., Zhu, T. R., and Welch, R. M.: Clustering, randomness and regularity in cloud fields .1. Theoretical considerations, J. Geophys. Res.-Atmos., 97, 20519-20536, 1992.

Weger, R. C., Lee, J., and Welch, R. M.: Clustering, randomness, and regularity in-cloud fields .3. The nature and distribution of clusters, J. Geophys. Res.-Atmos., 98, 18449-18463, 1993.

Wen, G. Y., Marshak, A., Cahalan, R. F., Remer, L. A., and Kleidman, R. G.: 3-D aerosol-cloud radiative interaction observed in collocated MODIS and ASTER images of cumulus cloud fields, J. Geophys. Res.-Atmos., 112, D13204, doi:10.1029/2006jd008267, 2007.
Wen, G. Y., Marshak, A., and Cahalan, R. F.: Importance of molecular Rayleigh scattering in the enhancement of clear sky reflectance in the vicinity of boundary layer cumulus clouds, J. Geophys. Res.-Atmos., 113(10), D24207, doi:10.1029/2008jd010592, 2008.

Zhu, T., Lee, J., Weger, R. C., and Welch, R. M.: Clustering, randomness, and regularity in cloud fields .2. Cumulus cloud fields, J. Geophys. Res.-Atmos., 97, 20537-20558, 1992.

Zinner, T., Marshak, A., Lang, S., Martins, J. V., and Mayer, B.: Remote sensing of cloud sides of deep convection: towards a three-dimensional retrieval of cloud particle size profiles, Atmos. Chem. Phys., 8, 4741-4757, doi:10.5194/acp-8-4741-2008, 2008. 